\title{
Nonlinear Grey-Box Identification of Linear Actuators Containing Hysteresis
}

Johan Gunnar, Erik Wernholt, Geir Hovland, Torgny Brogårdh

\author{
Division of Automatic Control \\ Department of Electrical Engineering \\ Linköpings universitet, SE-581 83 Linköping, Sweden \\ WWW: http://wWw. control.isy.liu.se \\ E-mail: johgu848@student.liu.se, erikw@isy.liu.se, \\ hovland@itee.uq.edu.au, torgny.brogardh@se.abb.com
}

29th August 2006

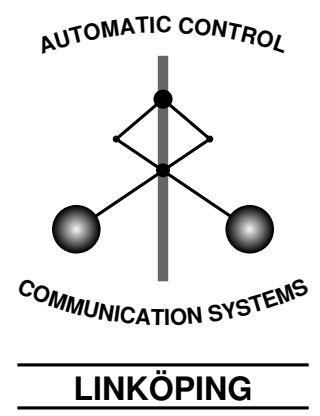

Report no.: LiTH-ISY-R-2741

Accepted for publication in ICRA 2006, Orlando, Florida

Technical reports from the Control \& Communication group in Linköping are available at http://www.control.isy.liu.se/publications. 


\begin{abstract}
A new identification procedure for a linear actuator used in parallel kinematic manipulators has been developed. The actuator dynamics contain both hysteresis and backlash resulting in a highly nonlinear system. The results in this paper show that not only can a nonlinear model of the system be successfully identified from measurement data, but the model is also compact enough to be an ideal candidate for inclusion in a high-performance robot control system.
\end{abstract}

Keywords: Identification, Robotics, Backlash, Hysteresis, Parallel kinematic manipulators 


\section{Nonlinear Grey-Box Identification of Linear Actuators Containing Hysteresis}

\author{
Johan Gunnar and \\ Erik Wernholt \\ Department of Electrical Engineering \\ Linköping University \\ SE-581 83 Linköping, Sweden \\ Email: johgu848@student.liu.se, \\ erikw@isy.liu.se
}

\author{
Geir Hovland \\ Information Technology and \\ Electrical Engineering \\ The University of Queensland \\ Brisbane, Australia QLD 4072 \\ Email: hovland@itee.uq.edu.au
}

\author{
Torgny Brogårdh \\ ABB Automation Technologies, Robotics \\ Hydrovägen 10, SE-721 68 Västerås, Sweden \\ Email: torgny.brogardh@se.abb.com
}

\begin{abstract}
A new identification procedure for a linear actuator used in parallel kinematic manipulators has been developed. The actuator dynamics contain both hysteresis and backlash resulting in a highly nonlinear system. The results in this paper show that not only can a nonlinear model of the system be successfully identified from measurement data, but the model is also compact enough to be an ideal candidate for inclusion in a high-performance robot control system.
\end{abstract}

\section{INTRODUCTION}

System identification in robotics is a vast research area and can be divided into, at least, three different levels or application areas. These levels involve the estimation of the kinematic description, the dynamic model (often divided into rigid body and flexible body dynamics), and the joint model (e.g., motor inertia, gear box elasticity and backlash, motor characteristics, and friction parameters). An overview of identification in robotics can also be found in [1].

In the work reported here the dynamics of the linear actuator of the Gantry-Tau Parallel Manipulator is investigated, including analysis and modeling of nonlinear hysteresis. The Tau family of parallel kinematic manipulators (PKMs) was introduced by ABB Robotics, see [2], [3], [4]. Other examples of PKMs utilizing linear actuators are the Hexaglide [5], the Triaglide [6] and the I4 [7], [8]. One of the main benefits of PKMs is the high stiffness of the arm structures. However, the overall stiffness of the PKM depends not only on the arm structure, but also on the actuators and the support structure. The work presented in this paper is a first step towards a PKM control system taking nonlinear actuator flexibilities into account.

The hysteresis model considered in this paper was originally presented in [9]. The method for estimation of the parameters in [9] is here used in the initial steps of the identification procedure. The gain of applying nonlinear grey-box identification on the model parameters is investigated. A three-step approach inspired by the method developed in [10] is applied. The procedure proposed in [10] is used to identify parameters for rigid body dynamics, friction, and flexibilities in a twomass model of an industrial serial-type robot. The first two steps find good initial parameter estimates for the iterative optimization routine, while the main step is the last, where the parameters of a nonlinear physically parameterized model (a nonlinear grey-box model) are identified directly in the time domain. In the work presented here the procedure in [10] has been modified to suit the identification of a linear actuator containing hysteresis.

Backlash in drive trains have been studied before, e.g. in [11] where grey-box identification of a two-mass model with backlash was considered. Black-box modeling was used to find initial parameter values. In [12] identification of hysteresis with methods based on an augmented EKF-filter was considered.

\section{PhysiCAL SYSTEM}

The physical system that is studied in this work is the linear actuator of a prototype of the Gantry-Tau robot. The actuator is made up of an AC-motor and a drive train, see Figure 1. The

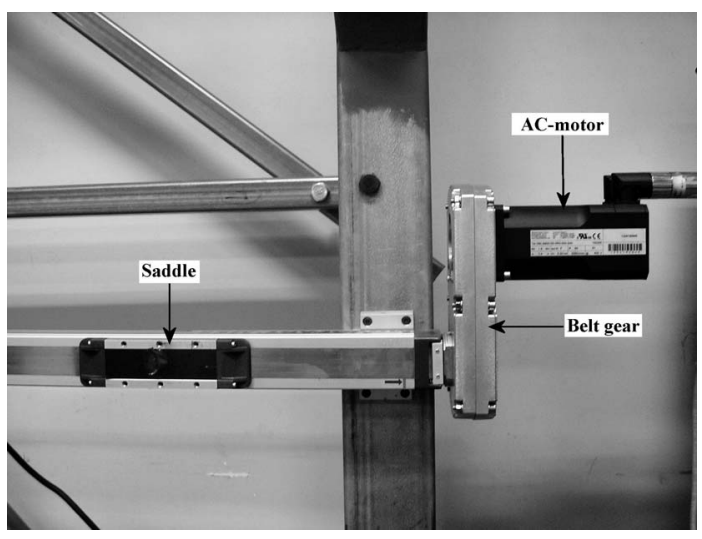

Fig. 1. The linear actuator on Gantry-Tau.

drive train consists of two parts, first a belt gear that connects the motor to a lead screw and then a nut that connects the screw to the saddle. The drive train transforms the rotation of the motor axis to a translative movement of the saddle. The Tau-structure is connected to the three actuators via the saddles. More detailed information about the actuators can be found in [13]. 


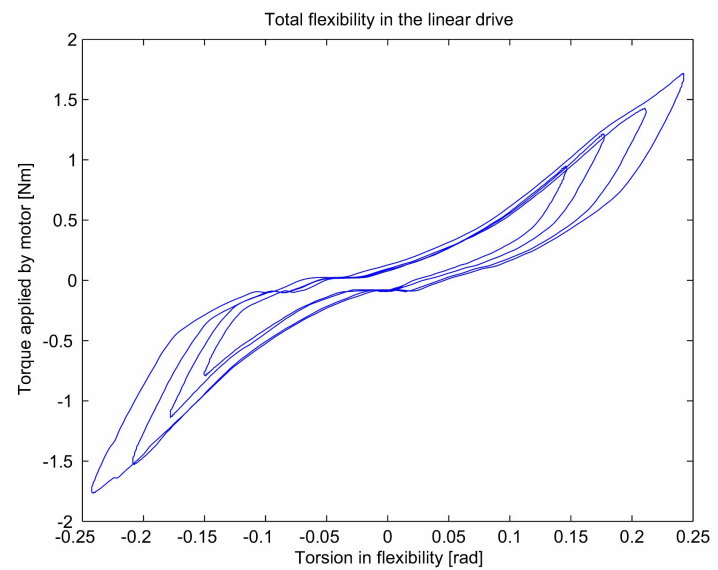

Fig. 2. Torque as a function of torsion. Four sinusoidal signals with different amplitudes applied as torsion of the flexibility.

Analysis of the system has showed that both the belt gear and the lead screw combined with the nut suffer from nonlinear characteristics and hysteresis [14]. In Figure 2 the effect of the flexibilities between the motor and the saddle is illustrated. Some of the nonlinear effects in the belt gear can be understood by studying the construction of the actuator. The belt that connects the two gear wheels is not fully stretched when not exposed to torque. This introduces backlash in the system and hence trying to analyze the flexibilities between the belt gear and the saddle is not easy. Analysis has however showed that the main flexibility is located in the lead screw and the nut. This also means that the stiffness of the system will depend on the position of the saddle. The stiffness is on the other hand also the result of torsion in the structure that supports the saddle. The stiffness will therefore not only depend on the position of the saddle, but also on the distance to the nearest support structure element. In this work all experiments were performed with the saddle at the same position to avoid these effects.

\section{NONLINEAR GREY-BOX IDENTIFICATION}

In the work presented here a beta version of a nonlinear extension to the System Identification Toolbox (SITB), [15], for Matlab will be used for the identification process. The extension contains a nonlinear grey-box model structure nlgrey which has similar properties as the idgrey structure. In the current version, only output error (OE) models can be used, i.e, models with only additive white noise on the output. The model will be described by a continuous-time state space structure

$$
\begin{aligned}
& \dot{x}(t)=f(t, x(t), \theta, u(t)) \\
& y(t)=h(t, x(t), \theta, u(t))+e(t)
\end{aligned}
$$

where both $f$ and $h$ are nonlinear functions, $x(t)$ is the state vector, $u(t)$ are the input signal and $y(t)$ are the output signal. $e(t)$ is white measurement noise and $\theta$ is the unknown parameter vector. The aim of the identification is to find the parameters that given data from the physical system will minimize the criterion

$$
V(\theta)=\frac{1}{N} \sum_{t=1}^{N} \epsilon^{2}(t, \theta)
$$

where $\epsilon(t, \theta)$ is the prediction error $\epsilon(t, \theta)=y(t)-\hat{y}(t, \theta)$. The prediction $\hat{y}(t, \theta)$ then becomes the simulated output of the model (1) with the input $u(t)$ (without $e(t)$ ). The toolbox estimates the parameter vector $\theta$ by applying a prediction error method, which performs a numerical optimization of the criterion. The numerical optimization is performed by an iterative numerical search algorithm.

\section{MODEL}

The dynamics of the system is approximated by a model consisting of two masses connected via a gear, a damper and a spring as shown in Figure 3. All flexibilities between the motor and the saddle, known to contain nonlinear effects, such as hysteresis and backlash, are lumped together and modeled as one flexibility.

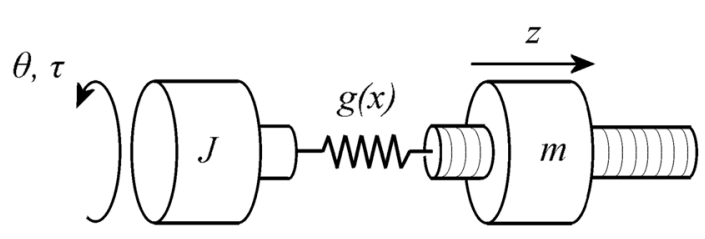

Fig. 3. Two-mass flexible model.

The input of the model is the torque, $\tau$, generated by the AC-motor and the output is the torsion in the flexibility, $\theta(t)-$ $z(t) / r$. The physical parameters of the model are defined as:

$$
\begin{array}{ll}
J & \text { moment of inertia }\left[\mathrm{Nms}^{2}\right] \\
m & \text { mass of saddle }[\mathrm{kg}] \\
F_{v} & \text { viscous friction parameter }[\mathrm{Nm} / \mathrm{s}] \\
F_{c} & \text { Coulomb friction parameter }[\mathrm{Nm}] \\
r & \text { gear ratio }[\mathrm{m} / \mathrm{rad}]
\end{array}
$$

The first mass in the model represents the rotating part of the electrical motor but also all rotating parts between the motor and the saddle, i.e, gear wheels, the belt and the lead screw. This is motivated by looking at where the main flexibility of the system is located. Analysis has showed that it is located in the lead screw and the nut which means that most of the moment of inertia between the motor and the saddle will be located at the motor side of the flexibility. This is however a significant approximation since some of the flexibility is located in the belt gear. The second mass in the model represents the saddle. Since the gear ratio is large, all friction is modeled at the motor side and the friction at the saddle is neglected. 
Introducing the states $x(t)^{T}=[\theta(t)-z(t) / r, \dot{\theta}(t), \dot{z}(t)]$, where $\theta(t)$ is the angle of the motor axis and $z(t)$ is the position of the saddle, and applying torque balances for the two masses gives the dynamic model

$$
\begin{aligned}
& \dot{x}_{1}=x_{2}-x_{3} / r \\
& \dot{x}_{2}=\frac{1}{J}\left(-F_{v} x_{2}-F_{c} \operatorname{sign}\left(x_{2}\right)-g(x)+\tau\right) \\
& \dot{x}_{3}=\frac{g(x)}{r m}
\end{aligned}
$$

The function $g(x)$ is the torque of the flexibility as a function of the states. A simple hysteresis model is added to approximate the nonlinear flexibility. The model was first used in [9], where hysteresis in a harmonic drive was studied. The hysteresis model has been chosen partly because of its simplicity. Since the model only contains four free parameters, $k_{1}$, $k_{2}, \alpha$ and $A$, it is an ideal candidate for use in identification. The introduction of the hysteresis model will add one extra state $x_{4}$, given by the state equation

$$
\dot{x}_{4}=-\alpha\left|x_{2}-x_{3} / r\right| x_{4}+A\left(x_{2}-x_{3} / r\right)
$$

The state $x_{4}$ corresponds to the nonlinear friction within the flexibility. The state takes account of the whole torque history and adds the memory of the hysteresis to the model. Statically the friction torque $x_{4}$ corresponds to a Coulomb-type friction, $x_{4}=\frac{A}{\alpha} \operatorname{sign}\left(x_{2}-x_{3} / r\right)$.

The torque function will be modeled as

$$
g(x)=k_{1} x_{1}+k_{2} x_{1}^{3}+x_{4}
$$

where $k_{1} x_{1}+k_{2} x_{1}^{3}$ models the stiffness torque of the flexibility.

While studying the ability to describe the nonlinear characteristics with different orders of odd polynomial functions of $x_{1}$ in $g(x)$, using step 2 in Section $\mathrm{V}$, no significant improvement was noticed between a function with two, and one with three terms. Therefore a lower order of polynomial function in $g(x)$ is chosen compared to [9]. The choice of a lower order function is also done to reduce the number of parameters in the model.

Since the hysteresis model only introduces one extra state, the model is, although describing some complicated nonlinear effects, very compact. This makes it an ideal candidate for inclusion in a control system.

\section{IDENTIFICATION PROCEDURE}

The aim of the identification is to estimate all the parameters in the model using nonlinear grey-box identification procedures. A problem of iterative search methods for solving nonlinear least squares problems is that only local convergence can be guaranteed. This means that good initial values of all parameters in the model are essential to achieve successful identification. The problem has been studied before, e.g. in [10] where a three-step method for the identification of a nonlinear model for industrial robots was proposed. Inspired by the results in [10] a three-step method to identify a model for the system is proposed here as well, although only the first step is identical to the earlier proposed procedure. The main difference is that here the second mass is fixed in steps 2 and 3. The reason for this is to enable an easier identification of the parameters in the flexibility. There are two advantages of constructing an experiment in this way and not try to identify the parameters through an experiment with the saddle able to move. First, it solves the problem of finding initial values for the hysteresis model since the procedure in [9] can be used. Secondly, it makes it easier to excite the flexibility enough to accurately identify the parameters. The mass in the saddle is so small, that without an extra load it would be very hard to excite the system enough to identify all parameters in the model through a single experiment. Since the load-carrying capability of the actuator is limited, adding a heavy weight was no alternative.

In the proposed procedure the first step initiates the parameters for the rigid body dynamics solving a least squares problem. In the second step the hysteresis model is initiated solving a nonlinear least squares problem. Finally, in the third step, the procedures for nonlinear grey-box identification are applied.

\section{A. Step 1. Initial values for rigid body dynamics and friction}

In the first step an experiment to identify the parameters of the rigid body dynamics is constructed with the saddle able to move. The rigid body dynamics of the system are described by

$$
J_{t} \ddot{\theta}+F_{v} \dot{\theta}+F_{c} \operatorname{sign}(\dot{\theta})=\tau
$$

where $\theta$ is the angle of the motor axis, $F_{c}$ is the Coulomb friction, $F_{v}$ is the viscous friction. $J_{t}$ is the total moment of inertia of the system. This means that $J_{t}=J+r^{2} m$. Rewriting (6) as a linear regression gives

$$
\left(\begin{array}{lll}
\ddot{\theta} & \dot{\theta} & \operatorname{sign}(\dot{\theta})
\end{array}\right)\left(\begin{array}{c}
J+r^{2} m \\
F_{v} \\
F_{c}
\end{array}\right)=\tau
$$

The parameters can now be estimated by solving (7) as a standard least-squares problem.

\section{B. Step 2. Initial values for the hysteresis model}

While several methods for initiating parameters for springs and dampers in linear systems have been studied before, these are of little help when identifying a nonlinear model with a strong hysteresis. In [10] a method based on a linear approximation and the use of an estimated Frequency Response Function (FRF) was used for initiating a nonlinear spring. As mentioned before the system studied here is very hard to excite when the mass of the saddle is small and the gear ratio large. This, in combination with the fact that the system suffers from hysteresis and a significant nonlinearity rules out the use of the method in [10]. Instead the procedure derived in [9] is used to initiate the parameters of the hysteresis model. The procedure is based on bringing the hysteresis into a steadystate loop while the second mass is fixed. If the second mass 
is fixed, then $x_{3}=0$ and the system is described by

$$
\begin{aligned}
\dot{x}_{1} & =x_{2} \\
\dot{x}_{2} & =\frac{1}{J}\left(-F_{v} x_{2}-F_{c} \operatorname{sign}\left(x_{2}\right)-g(x)+\tau\right) \\
\dot{x}_{4} & =-\alpha\left|x_{2}\right| x_{4}+A x_{2} \\
g(x) & =k_{1} x_{1}+k_{2} x_{1}^{3}+x_{4}
\end{aligned}
$$

This will be the model used for steps 2 and 3 .

The steady-state hysteresis loop is described by the stiffness curve $\phi\left(x_{1}\right)=k_{1} x_{1}+k_{2} x_{1}^{3}$, one ascending curve $C_{u}\left(x_{1}\right)$ for $\dot{x}_{1}>0$ and one descending curve $C_{d}\left(x_{1}\right)$ for $\dot{x}_{1}<0$. For an excitation signal $x_{1}=Q_{j} \sin (w t)$ the curves are derived in [9] as

$$
\begin{aligned}
C_{u j}\left(x_{1}\right) & =-\frac{A}{\alpha}\left(-1+2 \frac{e^{\alpha\left(Q_{j}+x_{1}(t)\right)}}{1+e^{-2 \alpha Q_{j}}}\right) \\
C_{d j}\left(x_{1}\right) & =\frac{A}{\alpha}\left(-1+2 \frac{e^{\alpha\left(Q_{j}-x_{1}(t)\right)}}{1+e^{-2 \alpha Q_{j}}}\right)
\end{aligned}
$$

Using the derived functions and measuring the torque while exciting the system so that $x_{1}=Q_{j} \sin (w t)$ the parameters are estimated as the minimizing arguments to the nonlinear least-squares problem

$$
F=\sum_{j=1}^{m} \sum_{i=1}^{n}\left(\epsilon_{u j}(i)^{2}+\epsilon_{d j}(i)^{2}\right)
$$

where $\epsilon_{k j}(i)=\phi\left(x_{1 j}(i)\right)+C_{k j}\left(x_{1 j}(i)\right)-\tau_{n j}(i)$ are the model equation errors corresponding to the hysteresis loop $j$ and the data point $i$ for the ascending and descending curve, respectively. $m$ is the number of loops and $n$ is the number of data points. $\tau_{n}$ is the torque on the flexibilities, that is $\tau_{n}=\tau-F_{v} x_{2}-F_{c} \operatorname{sign}\left(x_{2}\right)$.

\section{Step 3. Nonlinear grey-box identification}

In the third step the nonlinear grey-box identification method is applied. The hysteresis parameters are estimated using data achieved with the saddle fixed and the model defined by (8).

\section{DATA COLLECTION}

The equipment used for the experiments makes it possible to collect the data for the identification experiments only while the control system is in use. Since the control system is not fully known to the user, the only way to use the closedloop data is for so called direct identification, i.e., the data is used without any assumption about how it was generated. The estimates found in this way might get biased as an effect of the feedback in the system [16]. The equipment does however make it possible to give a reference either as speed or as torque to the control system. With a speed reference one can easily construct the experiments so that the saddle does not reach the endpoints of the linear drive and it is a suitable method to use when the saddle is free. The main limitation is that the control system is unable to follow very small variations of the motor axis, which is needed for identifying the flexibility. The system can more easily follow a torque reference and therefore most of the experiments will be performed in this way.

\section{A. Experiment design}

While applying the identification procedure described above there are needs for several different excitation signals. In step 1 where the parameters in the rigid body dynamics are identified one wishes to excite those parameters without exciting the flexibility. To be able to do this a signal with low frequency is chosen for the excitation. In step 2 the method is based on an excitation signal that brings the system to a state where $x_{1}=Q_{j} \sin (w t)$, for several different amplitudes $Q_{j}$. Since $x_{1}$ is measured, the desired excitation signal can be obtained by using a simple controller and a sinusoidal signal as reference. In step 3 the whole frequency band where the model is supposed to be used should be excited as well as all the parameters in the model. In this case this means that frequencies up to at least $25 \mathrm{~Hz}$ should be excited.

Eight different data sets were used for the identification and validation. One was collected with the saddle free to move and with the signal as a reference speed for the controller.

- Data set 1: Sinusoidal signal. $10 \mathrm{~s}$ of data, with amplitude $210 \mathrm{rad} / \mathrm{s}$ and frequency of $1 \mathrm{~Hz}$.

Seven data sets were collected with the saddle fixed. Data sets 2-5 were collected for the initiation of the hysteresis model. A PI-controller was used to make the motor angle follow the reference signal. Data sets 6-8 were collected with the signal as torque reference for the controller.

- Data sets 2-5: Sinusoidal signals with different amplitude as reference angle. Angular velocity $0.5 \mathrm{rad} / \mathrm{s}, 12.3 \mathrm{~s}$ data. The amplitudes of the sinusoidal motion in these data sets have been chosen so that the maximum torque applied to the flexibility is in the interval between 0.9 and $1.6 \mathrm{Nm}$.

- Data set 6: Sinusoidal signal with an angular velocity of $2 \mathrm{rad} / \mathrm{s}$ and an amplitude of $1.4 \mathrm{Nm}$.

- Data sets 7-8: Multi-sine signals (sum of sinusoids), $5 \mathrm{~s}$ of data, frequencies in the interval $1-30 \mathrm{~Hz}$. Two different sets with different amplitude and phase were collected.

For details on the selection of excitation signals, see, e.g. [16], [17].

\section{B. Preprocessing of data}

In step 1 of the identification procedure the motor angular velocity and the angular acceleration are used. They are derived through numerical differentiation of the measured motor angle. All data are low-pass filtered to avoid high frequency noise. For this purpose a fourth order Butterworth filter with cut-off frequency $100 \mathrm{~Hz}$ was used together with the Matlab command filtfilt. In some data sets the sample means have also been removed before filtering the signal.

\section{RESULTS}

The three-step method described above has been applied to the data sets to identify the physical parameters in the actuator model. The gear ratio $\mathrm{r}$ is know to be $r=1.867 \cdot 10^{-3} \mathrm{~m} / \mathrm{rad}$ and the mass of the saddle is known to be $m=1.2 \mathrm{~kg}$. 


\section{A. Step 1}

Solving the least-squares problem formed by (7) and data set 1 gives the following parameter estimates including one standard deviation

$$
\left(\begin{array}{c}
J+r^{2} m \\
F_{v} \\
F_{c}
\end{array}\right)=\left(\begin{array}{l}
5.437( \pm 0.083) \cdot 10^{-4} \\
1.330( \pm 0.140) \cdot 10^{-3} \\
2.872( \pm 0.210) \cdot 10^{-1}
\end{array}\right)
$$

The rather large standard deviation indicates that the model cannot fully describe the data set. This could mean that a more advanced friction model than the one used here must be applied to describe the data accurately. It can also mean that the flexibility, although not meant to, is excited in the data set so that the data cannot be modeled accurately with rigid body dynamics. Since the mass of the saddle is known the moment of inertia can be calculated. This gives $J=5.396 \cdot 10^{-4} \mathrm{Nms}^{2}$.

\section{B. Step 2}

The methods for initiating the hysteresis model has been applied to data sets 2 to 5. The amplitudes of the sinusoidal motion in these data sets have been chosen so that the maximum torque applied to the flexibility is in the interval between 0.9 and $1.6 \mathrm{Nm}$. The hysteresis loops can be seen in Figure 2. Each data set contains one period of data chosen at a time when the system has converged to a steady-state loop. The nonlinear least-squares problem is solved with the lsqnonlin command in Matlab. Fifty iterations give the following parameter estimates

$$
\left(\begin{array}{c}
k_{1} \\
k_{2} \\
\alpha \\
A
\end{array}\right)=\left(\begin{array}{c}
1.104 \cdot 10^{1} \\
1.768 \cdot 10^{2} \\
7.192 \cdot 10^{1} \\
6.149 \cdot 10^{1}
\end{array}\right)
$$

Since the angular velocities in the data set are small the viscous friction was neglected when the parameters were estimated, using $\tau_{n} \approx \tau-F_{c} \operatorname{sign}\left(x_{2}\right)$.

\section{Step 3}

With the initial parameters derived in steps 1 and 2, resulting in model mo defined by (8), the methods for nonlinear grey-box identification were now applied to the model. The optimization was terminated after thirty iterations or when the expected improvement in the loss function was less then $0.01 \%$. For estimating the parameters, data set 6 was used combined with data sets 7 or 8 as shown in Table I. Data set 6 is included to stabilize the estimation of the parameters in the force function. Two models where the rigid body dynamics are kept fixed during the identification are also presented. The quality of the models is assessed using the model fit, defined in [16] as

$$
f i t=100\left(1-\frac{\sqrt{\sum_{t=1}^{N}(y(t)-\hat{y}(t))^{2}}}{\sqrt{\sum_{t=1}^{N}(y(t)-\bar{y})^{2}}}\right)
$$

where $y(t)$ is the measured output, $\hat{y}(t)$ is the predicted output and $\bar{y}$ is the mean value of $y(t)$.
TABLE I

ESTIMATION OF PARAMETERS, MO FROM STEPS $1 \& 2$, M 67 FROM STEP 3 USING DATA SETS 6 AND 7 AND M 68 FROM STEP 3 USING DATA SETS 6 AND 8. PARAMETER VALUES MARKED WITH * IN M67F AND m68F ARE KEPT FIXED DURING IDENTIFICATION.

\begin{tabular}{|c|c|c|c|c|c|c|}
\hline & $\mathrm{m} 0$ & $\mathrm{~m} 67 \mathrm{f}$ & $\mathrm{m} 68 \mathrm{f}$ & $\mathrm{m} 67$ & $\mathrm{~m} 68$ & \\
\hline$J$ & 5.396 & $5.396^{*}$ & $5.396^{*}$ & 4.991 & 5.659 & $\left(\cdot 10^{-4}\right)$ \\
\hline$F_{v}$ & 1.330 & $1.330^{*}$ & $1.330^{*}$ & 1.413 & 1.360 & $\left(\cdot 10^{-3}\right)$ \\
\hline$F_{c}$ & 2.872 & $2.872^{*}$ & $2.872^{*}$ & 2.065 & 2.614 & $\left(\cdot 10^{-1}\right)$ \\
\hline$k_{1}$ & 1.104 & 1.080 & 1.009 & 1.131 & 1.010 & $\left(\cdot 10^{1}\right)$ \\
\hline$k_{2}$ & 1.768 & 1.751 & 1.872 & 1.443 & 1.859 & $\left(\cdot 10^{2}\right)$ \\
\hline$\alpha$ & 7.192 & 10.113 & 9.493 & 11.250 & 10.207 & $\left(\cdot 10^{1}\right)$ \\
\hline $\mathrm{A}$ & 6.149 & 4.406 & 4.672 & 4.249 & 4.427 & $\left(\cdot 10^{1}\right)$ \\
\hline
\end{tabular}

TABLE II

MODEL FIT WHEN VALIDATING THE MODELS WITH ESTIMATED PARAMETERS ON DATA SETS 6, 7 AND 8.

\begin{tabular}{|c|c|c|c|c|c|}
\hline & $\mathrm{m} 0$ & $\mathrm{~m} 67 \mathrm{f}$ & $\mathrm{m} 68 \mathrm{f}$ & $\mathrm{m} 67$ & $\mathrm{~m} 68$ \\
\hline Data set 6 & 82.52 & 89.37 & 88.46 & 88.27 & 89.31 \\
\hline Data set 7 & 66.96 & 75.64 & 74.63 & 78.02 & 75.11 \\
\hline Data set 8 & 51.98 & 66.78 & 65.73 & 70.44 & 68.47 \\
\hline
\end{tabular}

The model fit, see Table II, is improved significantly through the nonlinear grey-box identification. The fit is increased from $83 \%$ to $88-89 \%$ for data set 6 , from $66 \%$ to $75-78 \%$ for data set 7 and from $52 \%$ to $66-70 \%$ for data set 8 . One can notice that data set 8 was generated with a similar signal as data set 7 , but with a smaller amplitude. It is probably the backlash in the system that makes is harder to predict data generated with a small amplitude. As seen by the increase in fit, this is where most is gained by applying nonlinear greybox identification. In Table I one can notice that the significant differences, compared to the estimates given by steps 1 and 2 , are in the parameters for the non-linear damping, $\alpha$ and $A$. Clearly the non-linear grey-box identification improves the parameter estimations compared to the initial ones. While the initialization method only optimizes the parameters with data from low frequency waves and only in a steady-state loop, the optimization in step 3 optimizes with data from a more complex signal that excite a broader band of frequencies.

To analyze the relative importance on the model fit for each parameter, the estimated model $\mathrm{m} 67$ is used and each parameter is perturbed $\pm 20 \%$, one at a time. In Table III the difference in model fit can be seen for the three data sets. It is clear that the parameters for the rigid body dynamics have small influence on the model fit. For data sets 6 and 8 the moment of inertia and the viscous friction hardly influence the fit at all, while they, probably due to higher velocities, have more effect on the fit for data set 7 . For this reason two models where these parameters are kept fixed are also presented. As seen in Table I this does not affect the estimates of the hysteresis parameters in a significant way. The poor influence of the parameters for the rigid body dynamics shows that, to get an accurate model, one has to find a way to 
TABLE III

DIFFERENCE IN MODEL FIT FOR M67, COMPARED TO TABLE II, WHEN EACH PARAMETER IS PERTURBED $+20 \% /-20 \%$ (ONE AT A TIME)

\begin{tabular}{|c|c|c|c|}
\hline & Data set 6 & Data set 7 & Data set 8 \\
\hline$J$ & $0.001 /-0.001$ & $-2.463 / 0.118$ & $-0.023 /-0.003$ \\
\hline$F_{v}$ & $-0.009 /-0.002$ & $0.116 / 0.120$ & $-0.040 / 0.002$ \\
\hline$F_{C}$ & $-0.124 / 0.013$ & $-0.101 / .150$ & $-1.087 / 0.201$ \\
\hline$k_{1}$ & $2.758 /-1.192$ & $-4.091 / 0.539$ & $-3.400 / 0.504$ \\
\hline$k_{2}$ & $2.139 /-2.000$ & $-0.333 / 0.631$ & $0.900 / 0.357$ \\
\hline$\alpha$ & $-2.357 /-1.943$ & $0.499 / 0.609$ & $1.844 /-0.047$ \\
\hline $\mathrm{A}$ & $-1.898 /-1.983$ & $-0.085 / 0.664$ & $-2.399 / 0.370$ \\
\hline
\end{tabular}

combine the method described here with experiments where these parameters are more excited.

In Figure 4 the measured and simulated torque is plotted against applied torque. This gives another validation of the fact that the estimated parameters in the hysteresis model actually models the flexibility satisfactorily. One can notice that even though the nonlinear grey-box identification has decreased the damping of the system compared to the initial values, the model has some problems to adapt to the data around zero torque. Since the backlash present in the system remains unmodeled in this work, it is believed to be one of the main reasons for these problems.

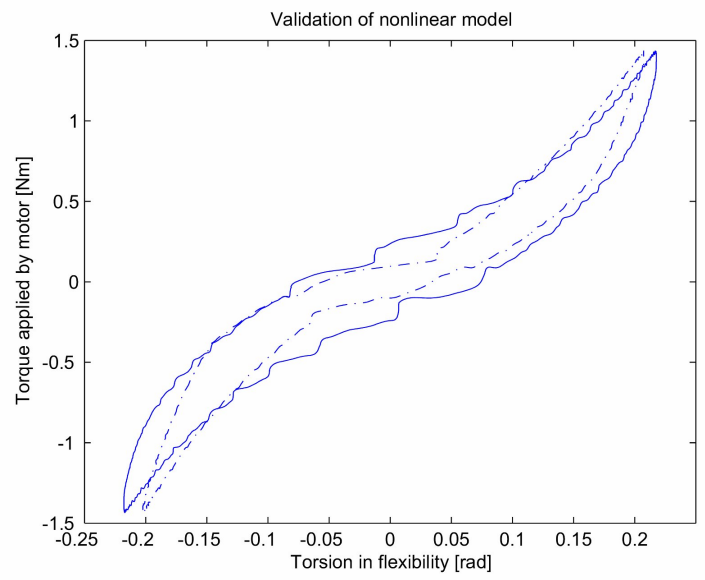

Fig. 4. Torque as a function of torsion. Dash-dotted curve is measured data, solid is simulated from model $\mathrm{m} 67$.

\section{CONCLUSIONS}

A three-step procedure to identify the rigid body dynamics, friction and hysteresis using only measurements on the motor side has been proposed and exemplified with real data. The work shows that the parameter estimates for the hysteresis model derived according to the common initialization steps can be improved using nonlinear grey-box identification. Especially the estimation of the nonlinear damping is significantly improved. The work also shows that although it is a simple model, the hysteresis model is able to model a great part of the behavior of the flexibility in the linear drive.
The relatively simple hysteresis model introduces one new dynamic state and hence is an ideal candidate for inclusion in a model-based PKM control system. Such a model-based controller will have its highest benefit for fast dynamic operations, such as pick-and-place.

There are several aspects of the work that are subjects for future studies. The poor excitation of the parameters in the rigid body dynamics is the most obvious one. An interesting topic for future work is to examine the possibilities to identify the system with the saddle free. This would open the possibility for better excitation of those parameters. Many problems are to be solved to make this possible. One is the problem with initiating the parameters in the hysteresis model. Another is to excite the system enough to be able to separate the masses and to make the hysteresis parameters identifiable.

\section{REFERENCES}

[1] K. Kozlowski, Modelling and identification in robotics, ser. Advances in Industrial Control. London: Springer, 1998.

[2] T. Brogårdh, "PKM research - important issues, as seen from a product development perspective at ABB robotics," in Proceedings of the Workshop on Fundamental Issues and Future Research Directions for Parallel Mechanisms and Manipulators, Quebec, Canada, Oct 2002.

[3] T. Brogårdh, S. Hanssen, and G. Hovland, "Application-oriented development of parallel kinematic manipulators with large workspace," in Proceedings of the 2nd International Colloquium of the Collaborative Research Center 562: Robotic Systems for Handling and Assembly, Braunschweig, Germany, May 2005, pp. 153-170.

[4] S. Johannesson, V. Berbyuk, and T. Brogårdh, "A new three degrees of freedom parallel manipulator," in Proceedings 4th Chemnitz Parallel Kinematics Seminar, Germany, Apr 2004, pp. 731-734.

[5] G. E. M. Honegger, A. Codourey, and E. Burdet, "Adaptive control of the hexaglide, a 6 dof parallel manipulator," in Proceedings 1997 IEEE Intl. Conf. on Robotics and Automation, Albuquerque, New Mexico, Apr 1997, pp. 543-548.

[6] L. Rey and R. Clavel, "The delta parallel robot," Parallel Kinematic Machines, C.R. Boër, L. Molinari-Tosatti, Springer, London, pp. 401418, 1999.

[7] S. Krut, O. Company, M. Benoit, H. Ota, and F. Pierrot, "I4: A new parallel mechanism for scara motion," in Proceedings of the 2003 IEEE International Conference on Robotics and Automation, Taipei, Taiwan, September 2003.

[8] P. Renaud, N. Andreff, S. Krut, and G. Gogu, "Kinematic calibration of linear-actuated parallel mechanisms from leg observation," in Proceedings of the 35th Intl. Symposium on Robotics, Paris, France, Apr 2004.

[9] R. Dhaouadi, F. H. Ghorbel, and P. S. Gandhi, "A new dynamic model of hysteresis in harmonic drives," IEEE Transactions on Industrial Electronics, vol. 50, no. 6, pp. 1165-1171, December 2003.

[10] E. Wernholt and S. Gunnarsson, "Nonlinear grey-box identification of industrial robots containing flexibilities," in Proc. 16th IFAC World Congress, Prague, Jul 2005.

[11] A. Isaksson, R. Lindkvist, X. Zhang, M. Nordin, and M. Tallfors, "Identification of mechanical parameters in drive train systems," in IFAC System Identification Symposium SYSID 2003, 2003.

[12] G. E. Hovland, S. Hanssen, E. Gallestey, S. Moberg, T. Brogårdh, S. Gunnarsson, and M. Isaksson, "Nonlinear identification of backlash in robot transmissions," in Proceedings of the 33rd ISR (International Symposium on Robotics), Oct 2002.

[13] Tollo, "Automation components - catalogue, part no: Dw110444gb," http://www.tollo.com/, Mars 2005.

[14] J. Gunnar, "Dynamical analysis and system identification of the gantry tau parallel manipulator," Master's thesis, Linköpings University, 2005.

[15] L. Ljung, System Identification Toolbox - User's Guide. Sherborn, MA, USA: The MathWorks Inc, 2003.

[16] - System Identification: Theory for the User, 2nd ed. Upper Saddle River, New Jersey, USA: Prentice Hall, 1999.

[17] R. Pintelon and J. Schoukens, System identification: a frequency domain approach. New York: IEEE Press, 2001. 


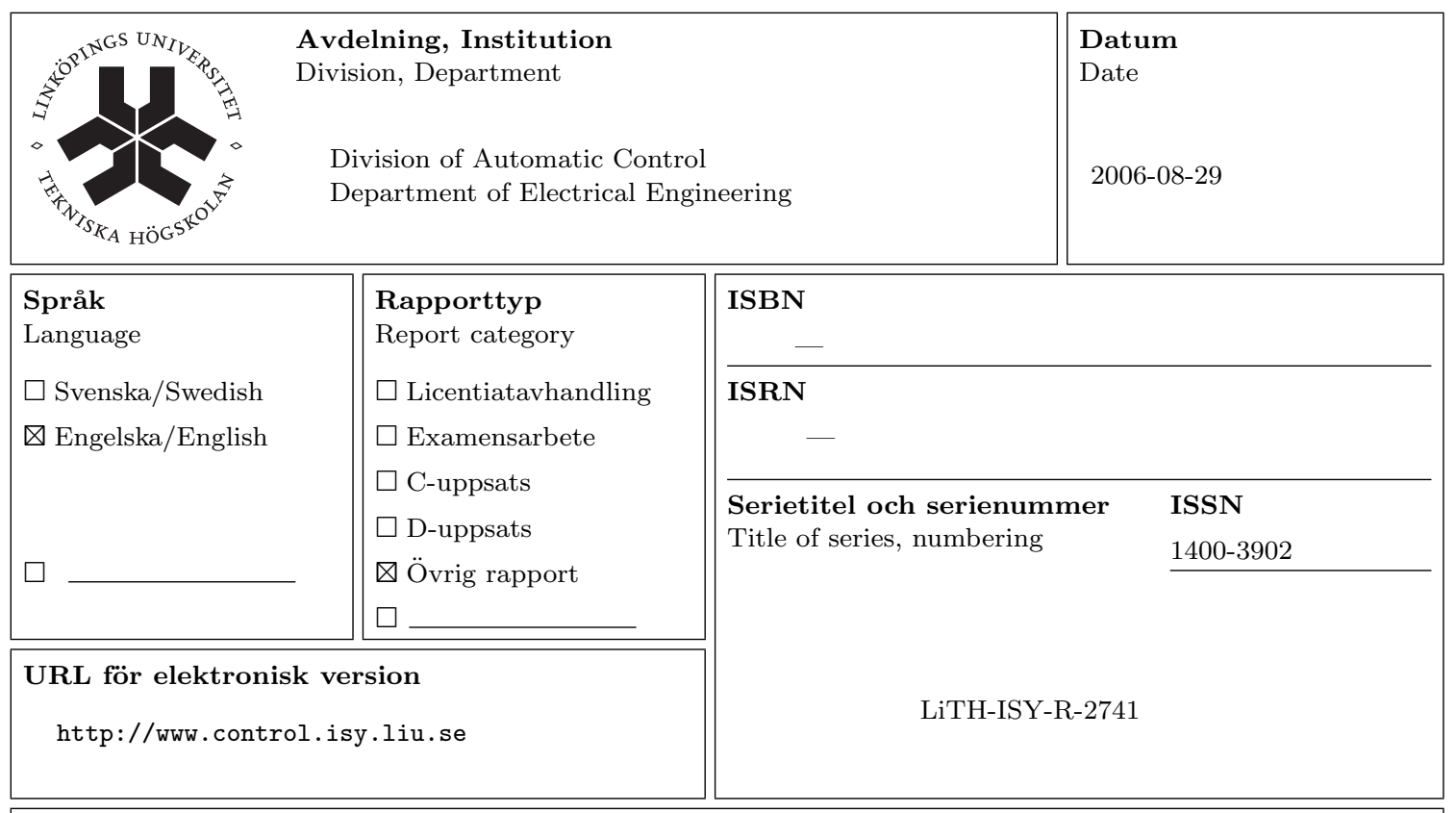

Titel Nonlinear Grey-Box Identification of Linear Actuators Containing Hysteresis

Title

Författare Johan Gunnar, Erik Wernholt, Geir Hovland, Torgny Brogårdh

Author

\section{Sammanfattning}

Abstract

A new identification procedure for a linear actuator used in parallel kinematic manipulators has been developed. The actuator dynamics contain both hysteresis and backlash resulting in a highly nonlinear system. The results in this paper show that not only can a nonlinear model of the system be successfully identified from measurement data, but the model is also compact enough to be an ideal candidate for inclusion in a high-performance robot control system. 